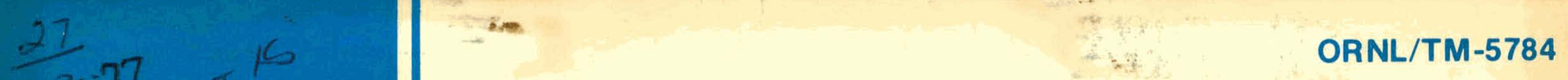

Calculation of a Self-Consistent, Low Frequency Electrostatic Field in Toroidal Geometry
C. O. Beasley
H. K. Meier
W. I. van Rij
J. E. McCune

MASTER 


\section{DISCLAIMER}

This report was prepared as an account of work sponsored by an agency of the United States Government. Neither the United States Government nor any agency Thereof, nor any of their employees, makes any warranty, express or implied, or assumes any legal liability or responsibility for the accuracy, completeness, or usefulness of any information, apparatus, product, or process disclosed, or represents that its use would not infringe privately owned rights. Reference herein to any specific commercial product, process, or service by trade name, trademark, manufacturer, or otherwise does not necessarily constitute or imply its endorsement, recommendation, or favoring by the United States Government or any agency thereof. The views and opinions of authors expressed herein do not necessarily state or reflect those of the United States Government or any agency thereof. 


\section{DISCLAIMER}

Portions of this document may be illegible in electronic image products. Images are produced from the best available original document. 

Printed in the United States of America. Available from National Technical Information Service
U.S. Department of Commerce
5285 Port Royal Road, Springfield, Virginia 22161
Price: Printen r.opy $\$ 4.00$; Microfiche $\$ 3.00$

This report was prepared as an account of work sponsored by the United States
Government. Neither the United States nor the Energy Research and Development
Administration/United States Nuclear Regulatory Commission, nor any of their
employees, nor any of their contractors, subcontractors, or their employees, makes
any warranty, express or implied, or assumes any legal liability or responsibility for the
accuracy, completeness or usefulness of any information, apparatus, product or
process disclosed, or represents that its use would not infringe privately owned rights. 
Contract No. W-7405-eng-26

FUSION ENERGY DIVISION

\title{
CALCULATION OF A SELF-CONSISTENT, LOW FREQUENCY ELECTROSTATIC FIELD IN TOROIDAL GEOMETRY
}

\author{
C. 0. Beasley \\ H. K. Meier \\ W. I. Van Rij \\ J. E. McCune \\ Massachusetts Institute of Technology \\ Cambridge, Massachusetts
}

\section{Date Published - March 1977}

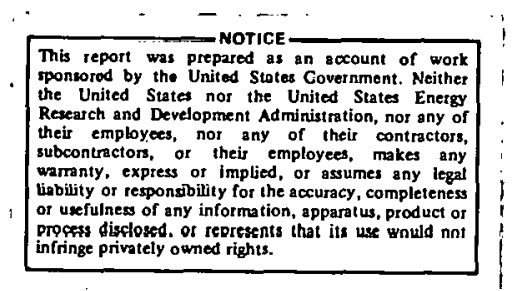

NOTICE This document contains information of a preliminary nature. It is subject to revision or correction and therefore does not represent a final report.

Prepared by the OAK RIDGE NATIONAI, I,ABORATORY

Oak Ridge, Tennessee operated by UNION CARBIDE CORPORATION for the ENERGY RESEARCH AND DEVELOPMENT ADMINISTRATION 
THIS PAGE WAS INTENTIONALLY LEFT BLANK 
TABLE OF CONTENTS

ABSTRACT ........................... v

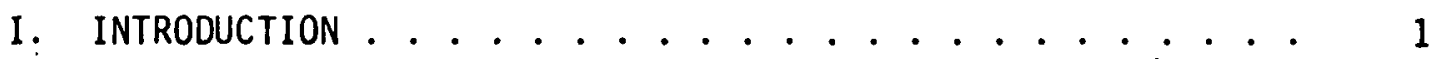

II. ASYMPTOTIC SERIES DERIVATION ............. 5

III. TOROIDAL GEOMETRY ................ 13

REFERENCES ............................. 17

APPENDIX. DRIFT-KINETIC APPROXIMATION ......... 19 


\section{THIS PAGE}

WAS INTENTIONALLY

LEFT BLANK 


\title{
CALCULATION OF A SELF-CONSISTENT, LOW FREQUENCY \\ ELECTROSTATIC FIELD IN TOROIDAL GEOMETRY*
}

C. 0. Beasley, H. K. Meier, W. I. $\operatorname{van} \mathrm{Rij}^{\dagger}$

Oak Ridge National Laboratory

Oak Ridge, Tennessee 37830

and

J. E. McCune

Massachusetts Institute of Technology

Cambridge, Massachusetts 02139

\begin{abstract}
We derive an asymptotic series in $\omega_{p}{ }^{-2}$, the inverse-square plasma frequency, for the self-consistent, curl-free, low frequency electric field in tori. The derivation is consistent with the drift-kinetic ordering and may be used in either instability or equilibrium calculations. We also provide an interpretation of past formulations of the statement of quasi-neutrality as applied to neoclassical equilibria.
\end{abstract}




\section{INTRODUCTION}

The creation of toroidally confined plasmas naturally results in the creation of spatial inhomogeneities in densities, currents, and temperatures, as well as in the confining magnetic field. These inhomogeneities drive species-dependent particle currents which cause charge separations, thus setting up electric fields. These electric fields in turn influence the currents, completing the cycle.

While the physically interesting phenomena relating to equilibrium or instability behavior of such plasmas occur on relatively long drift, collision, or bounce time scales, the mathematical coupling of the kinetic equations for the individual species and the electric field by use of Poisson's equation results in an extremely rapid oscillation at or near the plasma frequency $\omega_{p}$. Aside from the mathematical and numerical difficulties this oscillation introduces, it is of little physical interest in toroidal problems, and is also, of course, completely inconsistent with the drift-kinetic ordering used to describe the species kinematics in such problems.

To el iminate (or in effect average out) this rapid oscillation, the familiar concept of quasi-neutrality has been used. A number of applications appropriate to toroidal problems are discussed. ${ }^{1-3}$ The procedure involves a coupling which correctly models the low frequency response and at the same time precludes plasma oscillations. While relatively simple formulations used in the past have been suitable for analytic approximations, computation of equilibrium and instability (for instance, with the Collisional Plasma Mode ${ }^{4,5}$ ) necessitates a mathematically rigorous formulation consistent with the ordering 
used in the kinetic equation and an improved understanding of the concept of quasi-neutrality.

In treatments of trapped-particle instabilities, the formulation of quasi-neutrality (see, for example, Ref. 1) was used in dealing with a linearized instability in which the unperturbed distributions of electrons and ions were taken to be equal-density Maxwellians. To obtain a dispersion relation, it is almost always sufficient to state that the perturbed ion and electron densities are very nearly equal and to set $\tilde{N}_{i}=\tilde{N}_{e}$. In the process of obtaining the resulting dispersion relation, the electric field was eliminated from the problem, except for the integral operator

$$
\hat{K} \tilde{\phi} \equiv \frac{\oint \frac{d s}{v_{\|}} \tilde{\phi}}{\oint \frac{d s}{v_{\| 1}}}
$$

which was argued to be very nearly unity. Thus, good use was made of the notion of quasi-neutrality, but no discussion of its physical intcrpretation was ofrered.

For use in calculations of neoclassical transport, quasi-neutrality was formulated in more precise terms by Hazeltine and Hinton ${ }^{2}$ by setting

$$
\vec{\nabla} \cdot \vec{J}=\dot{U}
$$

where $\vec{j}$ is the tontal electric current density,

$$
\vec{j}=\sum_{s=\text { species }} e_{s} \vec{J}_{s}=\sum_{s} e_{s} N_{s} \vec{V}_{s} \text {, }
$$


and $e_{s}=Z_{s} e ; \vec{J}_{s}, N_{s}$ and $\vec{V}_{s}$ are the particle charges, fluxes, densities, and mass-flow velocities. By recognizing that most of the current is in the direction parallel to the magnetic field and imposing number and momentum conservation, one obtains

$$
\frac{\partial J}{\partial t}=\frac{Z e E N}{M}-\frac{\partial}{\partial z}\left(\frac{N k T}{M}\right)+V^{2} \frac{\partial N}{\partial z}+2 V \frac{\partial N}{\partial t},
$$

where $E$ is the electric field, $T$ the temperature, $M$ the mass, $k$ the Boltzmann constant, and the $z$ direction is parallel to the magnetic field. At equilibrium, $\dot{N}=j=0$; for a Maxwell-Boltzmann distribution,

$$
\text { ZeEN }=\frac{\partial}{\partial Z}(N k T),
$$

so that the expression used by Hazeltine and Hinton ${ }^{2}$ is obtained:

$$
\frac{\partial \rho}{\partial z}=0
$$

where $\rho=\sum_{s} e_{s} N_{s}$ i.s the charge density. Earlier, Hinton and Rosenbluth ${ }^{3}$ had used the flux=surface integral form of (I.1).

While the forms described above 2,3 eliminated the plasma oscillations and were suitable for obtaining the zeroth order electric field, the electric field is not obtained to an order in $\Omega^{-1}$ consistent with the drift-kinetic approximation. More specifically, the electric field resulting from perpendicular motion is ignored. Also, the formulation is not suitable for use in time-dependent problems. Finally, only the zeroth order in an $\omega_{p}^{-2}$ expansion is obtained, with no prescription for obtaining higher order corrections. 
In Sec. II, we obtain in a general geometry the proper ordering in $\omega_{p}{ }^{-2}$ for the electric field, charge, and current to first order in $\Omega^{-1}$. In Sec. III, we apply this formulation to a toroidal geometry. 


\section{ASYMPTOTIC SERIES DERIVATION}

The electric field consists of the externally applied ring field $\vec{E}_{r}$ and the plasma self-consistent field $\vec{E}$, which is the solution of Poisson's equation. An equation equivalent to Poisson's equation is the time-derivative equation

$$
\vec{\nabla} \cdot \frac{\partial^{2} \vec{E}}{\partial t^{2}}=4 \pi \frac{\partial^{2} \rho}{\partial t^{2}}
$$

coupled with the initial conditions

$$
\begin{aligned}
& \vec{\nabla} \cdot \vec{E}=4 \pi \rho]_{t=0}, \\
& {\left[\vec{\nabla} \cdot \frac{\partial \vec{E}}{\partial t}=4 \pi \frac{\partial \rho}{\partial t}\right]_{t=0} .}
\end{aligned}
$$

Charge continuity,

$$
\frac{\partial \rho}{\partial t}=-\vec{\nabla} \cdot \vec{j},
$$

can be used to eliminate $\rho$ from (II.1):

$$
\vec{\nabla} \cdot\left(\frac{\partial^{2} \vec{E}}{\partial t^{2}}+4 \pi \frac{\partial \vec{j}}{\partial t}\right)=0
$$

Further, the momentum-balance equation, multiplied by charge and summed over species, can be used to eliminate $\vec{j}$ from (II.4). This momentum equation can be written in the form

$$
4 \pi \frac{\partial \vec{j}}{\partial t}=\left(\omega_{p}^{2}\right) \vec{E}-\vec{G},
$$

where

$$
\omega_{p}^{2}=4 \pi \sum_{s} \frac{e_{s}{ }^{2} N_{s}}{M_{s}},
$$




$$
\begin{gathered}
\vec{G}=-\omega_{p}{ }^{2} \vec{E}_{r}-4 \pi \sum_{s} e_{s}\left[\left(\vec{J}_{s} \times \vec{\Omega}_{s}\right)+\int d^{3} v \vec{v} c_{s}-\vec{\nabla} \cdot \underline{k}_{s}\right], \\
\vec{s}_{s}=\frac{e_{s}}{\vec{M}_{s} c} \vec{B}, \\
\underline{\underline{K}}_{s}=\int d^{3} v \vec{v} \vec{v} f_{s},
\end{gathered}
$$

and $C_{S}$ is the collision operator. Clearly, the electron term will dominate the plasma frequency $\omega_{p}$ defined by (II.6).

The motivation for solving Poisson's equation in the form (II.4) is apparent from (II.5); it introduces the plasma frequency explicitly. However, in the drift-kinetic approximation, (II.5) is not satisfied because of the order mixing produced by the $\vec{J} \times \vec{\Omega}$ terms in (II.7). Therefore, we must calculate $(\partial \vec{j} / \partial t)$ from the drift-kinetic $\vec{j}$ using the drift-kinetic equation, if the ordering of the drift-kinetic approximation is to be preserved. Details of the implications of the drift-kinetic approximation for this purpose are given in the appendix for the case of a static magnetic field. We obtain

$$
4 \pi \frac{\partial \vec{j}}{\partial t}=\ddot{n}\left(\omega_{p}{ }^{2} \dot{E}_{\|}-\dot{G}_{0}\right)-\vec{G}_{1} \text {, }
$$

where

$$
\begin{aligned}
\hat{n} & =\frac{1}{B} \vec{B}, \\
E_{\|} & =\hat{n} \cdot \vec{E}, \\
G_{0}=-s_{p}{ }^{2} \hat{n} & =\vec{E}_{r}-4 \pi \sum_{s} c_{s} g_{O s}, \\
\vec{G}_{1} & =-4 \pi \sum_{s} e_{s} \vec{g}_{1 s} .
\end{aligned}
$$


The terms $g_{0}$ and $\vec{g}_{1}$ are fully defined in the appendix; here, it is sufficient to say that the 0 and 1 subscripts indicate the $\Omega^{-1}$ ordering and that $g_{0}$ has no explicit dependence on the self-consistent electric field $\vec{E}$.

We can now el iminate $(\partial \vec{j} / \partial t)$ from (II.4) by using (II.8), so that the equation to be solved is

$$
\vec{\nabla} \cdot\left(\frac{\partial^{2} \vec{E}}{\partial t^{2}}+\hat{n} \omega_{p}^{2} E_{\|}\right)=H_{0}+H_{1},
$$

where

$$
\begin{aligned}
& H_{0}=\vec{\nabla} \cdot\left(\hat{n} G_{0}\right), \\
& H_{1}=\vec{\nabla} \cdot \vec{G}_{1} .
\end{aligned}
$$

It is immediately obvious from (II.10) that any plasma oscillation effects in the perpendicular direction occur as a result of the $\vec{E}$ dependence in $H_{1}$ and are therefore reduced by a factor of order $\Omega^{-1}$ compared to the parallel plasma oscillation. This result simply reflects the familiar fact that, in devices with a strong magnetic field, particles can move much more freely along the field lines than perpendicular to them, so that charge separation and neutralization motions tend naturally to be in the parallel direction.

Appropriate to the assumed static magnetic field, the electrostatic case is taken,

$$
\vec{E}=-\vec{\nabla} \phi,
$$


and $\phi$ is separated into its ordered parts:

$$
\phi=\phi_{0}+\phi_{1}
$$

We now Fourier-analyze the $\phi_{m}$ and $H_{m}$ with respect to both position $\vec{x}$ and time $t$ :

$$
\begin{aligned}
& \phi_{m}(\vec{x}, t)=\int d^{3} k \int d \omega e^{i(\vec{k} \cdot \vec{x}+\omega t)} \phi_{m}(\vec{k}, \omega), \\
& H_{m}(\vec{x}, t)=\int d^{3} k \int d \omega e^{i(\vec{k} \cdot \vec{x}+\omega t)_{H_{m}}(\vec{k}, \omega)} .
\end{aligned}
$$

Equations (II.10), (II.12), (II.13), and (II.14) readily yield the relationship

$$
\left(k_{\|}^{2} \omega_{p}^{2}-k^{2} \omega^{2}\right) \phi_{m}(\vec{k}, \omega)=H_{m}\left(\vec{k}, \omega, \phi_{m-1}\right),
$$

where $\phi_{-1}=0$. In deriving (II.15), fluctuations in time and space of $\omega_{p}^{2}$ have been neglected, as has the variation of $B^{-1} \overrightarrow{V B}$ parallel to the magnetic fleld lines (since these approximations do not affect the content of the $\omega_{p}{ }^{-2}$ ordering). Equation (II.15) can be solved to give the time dependence of $\phi$, using the initial conditions (II.2):

$$
\begin{aligned}
k^{2} \phi_{m}(\vec{k}, t) & -4 \pi \dot{\omega}_{m}(\vec{k}, t)-P \int d \omega \frac{H_{m}\left(\vec{k}, \omega, \phi_{m-1}\right) e^{i \omega t}}{\tilde{\omega}_{p}^{2}-\omega^{2}} \\
+ & {\left[4 \pi \rho_{m}(\vec{k}, 0)-P \int d \omega \frac{H_{m}\left(\vec{k}, \omega, \phi_{m-1}\right)}{\tilde{\omega}_{p}^{2}-\omega^{2}}\right] \cos \tilde{\omega}_{p} t } \\
+ & \frac{1}{\tilde{\omega}_{p}}\left[4 \pi \dot{\rho}_{m}(\vec{k}, 0)-P \int d \omega \frac{\left.i \omega H_{m}(\vec{k}, \omega), \phi_{m-1}\right)}{\tilde{\omega}_{p}^{2}-\omega^{2}}\right] \sin \tilde{\omega}_{p} t,
\end{aligned}
$$


where

$$
\tilde{\omega}_{p}=\frac{k \|}{k} \omega_{p}
$$

The function $H_{0}(\vec{x}, t)$ is determined by the ring field, collision, and pressure terms [see (II.9a), (II.11a), and the appendix], and therefore will vary on a time scale that is much longer than $\omega_{p}{ }^{-1}$. Also, even the modified plasma frequency (II.17) is still very large. As an example, in toroidal devices, for all except flux-surface-averaged quantities (corresponding to $k_{\|}=0$ here and discussed in Sec. III), $\left|\frac{k}{k}\right| z \varepsilon$, the inverse aspect ratio. Consequently, we can obtain from (II.16) the low frequency (zerath order) portion of the potential in the form

$$
k^{2} \bar{\phi}_{0}(\vec{k}, t)=4 \pi \bar{\rho}_{0}(\vec{k}, t)=P \int d \omega \frac{H_{0}(\vec{k}, \omega) e^{i \omega t}}{\tilde{\omega}_{p}{ }^{2}-\omega^{2}},
$$

where $\bar{\phi}_{0}$ and $\bar{\rho}_{0}$ are in effect time averages over several modified plasma periods. Except for its dependence on the high frequency part of $\phi_{0}$, $H_{1}$ is also a low frequency function, and therefore the low frequency, first-order-correction potential is given by

$$
k^{2} \bar{\phi}_{1}(\vec{k}, t)=4 \pi \bar{\rho}_{1}(\vec{k}, t)=P \int d \omega \frac{H_{1}\left(\vec{k}_{, \omega}, \bar{\phi}_{0}\right) e^{i \omega t}}{\tilde{\omega}_{p}^{2}-\omega^{2}} .
$$

An asymptotic form exists for (II.18). Because the $H_{m}(\vec{x}, t)$ terms vary slowly in time, the numerators of the integrands will be vanishingly small unless $\left|\omega / \tilde{\omega}_{p}\right| \ll 1$, and consequently 


$$
\begin{gathered}
P \int d \omega \frac{H_{m}(\vec{k}, \omega) e^{i \omega t}}{\tilde{\omega}_{p}^{2}-\omega^{2}}=P \int d \omega\left[\sum_{j=0}^{N} \frac{\omega^{2 j}}{\tilde{\omega}_{p}^{2 j+2}}\right. \\
\left.+\frac{\omega^{2 N}}{\tilde{\omega}_{p}^{2 N+2}\left(\tilde{\omega}_{p}^{2}-\omega^{2}\right)}\right] H_{m}(\vec{k}, \omega) e^{i \omega t}=\sum_{j=0}^{N} \frac{(-1)^{j}}{\tilde{\omega}_{p}^{2 j+2}} \frac{\partial^{2 j}}{\partial t^{2 j}} H_{m}(\vec{k}, t)+R_{N}(\vec{k}, t),
\end{gathered}
$$

where the remainder $R_{N}$ will be negligibly small, provided $N$ is not too large. We therefore have

$$
k^{2} \bar{\phi}_{m}(\vec{k}, t)=4 \pi \bar{\rho}_{m}(\vec{k}, t)=\sum_{j=0}^{N} \frac{(-1)^{j}}{\hat{\omega}_{p}^{2 j+2}} \frac{\partial^{2 j}}{\partial t^{2 j}} \quad H_{m}(\vec{k}, t) .
$$

Differentiating (11.19) with respect to time, we get

$$
\begin{aligned}
& \vec{\nabla} \cdot \overline{\vec{j}}_{m}(\vec{k}, t)=-\dot{\bar{\rho}}_{m}(\vec{k}, t) \\
& =-\frac{1}{4 \pi} \sum_{j=0}^{N} \frac{(-1)^{j}}{\ddot{w}_{p}^{2 . j+2}} \frac{\partial^{2 . j+1}}{\partial L^{2 j+1}} H_{m}(\vec{k}, t):
\end{aligned}
$$

Solving Poisson's equation (II.19), we obtain $\bar{E}$ to order $(N+1)$ in $\omega_{p}{ }^{-2}$. It is then immediately apparent from (II.8) and (II.3) that the current and density calculated from the drift-kinetic equation will be determined only to order $\omega_{\mathrm{p}}^{-2 N}$. This illustrates explicitly how, with $N=0$, an electric field exists in an apparently neutral plasma (the plasma is "polarized"). Of course, (II.19) and (II.20) give the density and current to order $\omega_{p}^{-2(N+1)}$. 
For the application considered in Sec. III, we take $N=0$, and so it follows from (II.19), (II.17), (II.12), and (II.11) that the low frequency electrostatic field must satisfy the equations

$$
\begin{aligned}
& \vec{\nabla} \cdot\left[\hat{n}\left(\omega_{p}{ }^{2} \bar{E}_{\|, 0}-G_{0}\right)\right]=0, \\
& \vec{\nabla} \cdot\left[\hat{n} \omega_{p}{ }^{2} \bar{E}_{\|, 1}-\vec{G}_{1}\right]=0 .
\end{aligned}
$$

We can calculate $\vec{G}_{1}$ in (II.21b) with $\bar{E}_{0}=0$ because $\bar{E}_{0}$ would contribute a term of second order in $\omega_{p}{ }^{-2}$ to $\bar{E}_{\|, 1}$ and with $N=0$ such higher order terms are to be neglected. Equations (II.21a) and (II.21b) can be combined to give

$$
\vec{\nabla} \cdot\left[\hat{n}\left(\omega_{p}^{2} \bar{E}_{\|}-G_{0}\right)-\vec{G}_{1}\right]=0
$$

Comparing this with (II.10) and (II.11), we see that we can obtain the $(N=0)$ low frequency field by neglecting $\partial^{2} \vec{E} / \partial t^{2}$ in (II.10). However, a breakdown of (II.22) occurs for its flux-surface average. This problem is considered in Sec. III. 
THIS PAGE

WAS INTENTIONALLY

LEFT BLANK 


\section{TOROIDAL GEOMETRY}

We model the static magnetic field by the vacuum toroidal field and the plasma current poloidal field, taking care to keep $\vec{\nabla} \cdot \vec{B}=0$. In the usual toroidal geometry $\left(R_{0}=\right.$ major radius, $r=$ minor radius, $\theta=$ poloidal angle, $\zeta=$ toroidal angle), the model field is given by

$$
\vec{B}=\frac{\hat{n} B_{0}}{n(1+\varepsilon \cos \theta)}=\hat{n} B \text {, }
$$

where

$$
\begin{aligned}
\hat{n} & =\eta \delta \hat{\theta}+\eta \hat{\zeta}, \\
\eta & =\left(1+\delta^{2}\right)^{-1 / 2}, \\
\delta & =\frac{\varepsilon}{q(r)}, \varepsilon=\frac{r}{R_{0}} .
\end{aligned}
$$

If we flux-surface average (II.22), we obtain

$$
\frac{\partial}{\partial r} \quad\left[r<\hat{r} \cdot \vec{G}_{1}>\right]=0 .
$$

However, from the definition of $\vec{G}_{1}$ [see (II.9b) and the appendix] (III.2) is not satisfied in general. We must therefore use the exact equation (II.10) for the flux-surface average. We then obtain

$$
\frac{\partial}{\partial r}\left[r<\hat{r} \cdot\left(\frac{\partial^{2} \vec{E}}{\partial t^{2}}-\vec{G}_{1}\right)>\right]=0,
$$

a low frequency equation which relates $\langle\hat{r} \cdot \vec{E}\rangle$ to $\langle\hat{r} \cdot \vec{j}\rangle$ and corrects (III.2) by including the low frequency content of $\vec{E}$ in the flux average. We may thus obtain the complete low frequency electric field, including 
the flux-averaged part, by using (III.3). This implies using an improved version of (II.22):

$$
\vec{\nabla} \cdot\left[\hat{n}\left(\omega_{p}^{2} \bar{E}_{\|}-G_{\|}\right)-\vec{G}_{\perp}\right]=0,
$$

where

$$
\begin{aligned}
& G_{\|}=G_{0}+\hat{n} \cdot \vec{G}_{1}, \\
& \vec{G}_{\perp}=\vec{G}_{1}-\hat{n}\left(\hat{n} \cdot \vec{G}_{1}\right)-\hat{r}\left\langle\hat{r} \cdot \vec{G}_{1}\right\rangle .
\end{aligned}
$$

We may write (III.3) and (111.4) in terms of $\bar{\phi}$ by using (II.12)

and (III.1):

$$
\begin{gathered}
\frac{\partial}{\partial r}\left[r<\frac{\partial^{2}}{\partial t^{2}} \cdot \frac{\partial \Phi}{\partial r}+\hat{r} \cdot \vec{G}_{1}>\right]=0 \\
\frac{\partial^{2} \bar{\phi}}{\partial \theta^{2}}+\left(\frac{2 q}{1+\varepsilon \cos \theta}\right) \frac{\partial^{2} \bar{\phi}}{\partial \theta \partial \zeta}+\left[\frac{q \varepsilon \sin \theta \cdot}{(1+\varepsilon \cos \theta)^{2}}\right] \frac{\partial \bar{\phi}}{\partial \zeta}+\left[\frac{q^{2}}{(1+\varepsilon \cos \theta)^{2}}\right] \frac{\partial^{2} \bar{\phi}}{\partial \zeta^{2}} \\
=-\frac{1}{\left\langle\omega_{p}^{2}>\right.}\left(\frac{q R_{0}}{\eta}\right)^{2} \vec{\nabla} \cdot\left(\hat{n} G_{\|}+\vec{G}_{\perp}\right)
\end{gathered}
$$

Because $\bar{\phi}$ must satisfy toroidal periodicity, we can expand it in a Fourier series,

$$
\bar{\phi}=\underset{\mu \lambda}{\sum} \bar{\phi}_{\mu \lambda} \exp [i(\mu \theta-\lambda \zeta)],
$$

and solve (III.5) for all $\bar{\phi}_{\mu \lambda}$.

For the zeroth order potential we obtain the simpler equations

$$
\begin{gathered}
\frac{\partial}{\partial r}\left[r\left\langle\frac{\partial^{2} \bar{\phi}}{\partial t^{2}}\right\rangle\right]=0, \\
\frac{\partial \bar{\phi}}{\partial \theta}+\left(\frac{q}{1+\varepsilon \cos \theta}\right) \frac{\partial \bar{\phi}}{\partial \zeta}=\frac{q R_{0}}{n\left\langle\omega_{p}^{2}\right\rangle}\left(\frac{K(r)}{1+\varepsilon \cos \theta}-G_{0}\right),
\end{gathered}
$$


where the integration constant $K$ is given by

$$
K=\frac{\sqrt{1-\varepsilon^{2}}}{4 \pi^{2}} \oint d \theta \oint d \zeta G_{0} \text {. . }
$$

To this order, $[(\partial / \partial r)<r(\partial \bar{\phi} / \partial r)>]$ remains zero for an initially neutral plasma.

This result provides a general expression for the self-consistent electric field in a toroidal plasma to first order in $\Omega^{-1}$ and to lowest order in an $\omega_{\mathrm{p}}{ }^{-2}$ expansion. Unlike earlier treatments, this formulation is valid for any plasma distribution function: In the zeroth order limit in both the $\omega_{p}{ }^{-2}$ and $\Omega^{-1}$ expansions, it reduces to the form used by Hazeltine and Hinton ${ }^{2}$ for Maxwellian plasmas. We have also seen formally how one obtains the higher-order terms in the $\omega_{p}^{-2}$ expansion [see Eq. (II.19)]. 


\section{THIS PAGE}

\section{WAS INTENTIONALLY \\ LEFT BLANK}




\section{'REFERENCES}

* Research sponsored by the Energy Research and Development Administration under contract with Union Carbide Corporation.

† Computer Sciences Division.

1 B. B. Kadomtsev and 0. P. Pogutse, Dok. Akad. Nauk SSSR 186, 553 (1969) [Sov. Phys.-Doklady 14, 470 (1969)].

2 R. D. Hazeltine and F. L. Hinton, Phys. Fluids 16, 1883 (1973).

3 F. L. Hinton and M. N. Rosenbluth, Phys. Fluids 16, 386 (1973).

4 H. K. Mejer, W. I. van Rij, C. O. Beasley, Jr., and J. E. McCune, to be published in Plasma Physics.

5 W. I. van Rij, H. K. Meier, C. 0. Beasley, Jr., and J. E. McCune, to be published in Plasma Physics. 
THIS PAGE

WAS INTENTIONALLY

LEFT BLANK 
19

APPENDIX. DRIFT-KINETIC APPROXIMATION

The drift-kinetic equation is given by.

$$
\left(\frac{\partial}{\partial t}+\vec{V} \cdot \vec{\nabla}+\vec{A} \cdot \vec{\nabla}_{v}\right) \bar{f}=\bar{C},
$$

where

$$
\begin{gathered}
\vec{v}_{0}=\hat{n} v \cos \alpha, \\
\vec{v}_{1}=\frac{1}{\Omega}\left\{\vec{a} \times \hat{n}+\frac{1}{3}(\hat{n} \times \vec{b}+\vec{\nabla} \times \hat{n}) v^{2}\right. \\
\left.-\frac{1}{3}[\hat{n} \times \vec{b}+3 \hat{n}(\hat{n} \cdot \vec{\nabla} \times \hat{n})-2 \vec{\nabla} \times \hat{n}] v^{2} p_{2}(\cos \alpha)\right\} \\
\vec{A}_{0}=\hat{n}(\hat{n} \cdot \vec{a})+\frac{1}{2} \hat{\alpha}(\hat{n} \cdot \vec{b}) v^{2} \sin \alpha, \\
\vec{A}_{1}=\frac{1}{2 \Omega} \hat{s}[\hat{n} \times \vec{b}+\hat{n}(\hat{n} \cdot \vec{\nabla} \times \hat{n})] \cdot \vec{a} v \sin \alpha \\
+\frac{1}{\Omega} \hat{n}[\vec{\nabla} \times \hat{n}-\hat{n}(\hat{n} \cdot \vec{\nabla} \times \hat{n})] \cdot \vec{a} v \cos \alpha \\
-\frac{1}{2} \hat{\alpha} \vec{\nabla} \cdot\left\{\frac{1}{\Omega}[\vec{\nabla} \times \hat{n}-\hat{n}(\hat{n} \cdot \vec{\nabla} \times \hat{n})]\right\} v^{3} \sin \alpha \cdot \cos \alpha,
\end{gathered}
$$

and $\bar{C}$ is the collision operator. In the above,

$$
\hat{n}=\frac{1}{B} \vec{B}=\hat{v} \cos \alpha-\hat{\alpha} \sin \alpha \text {, }
$$


20

$$
\begin{aligned}
\hat{s}=\hat{v} \sin \alpha+\hat{\alpha} \cos \alpha, \\
\Omega=\frac{Z e B}{M c}, \vec{a}=\frac{Z e}{M}\left(\vec{E}+\vec{E}_{r}\right), \\
\vec{b}=\frac{1}{B} \vec{\nabla} B,
\end{aligned}
$$

and it is assumed that $\vec{B}$ is static. We have chosen the velocity-space coordinates described in Refs. 4 and 5 for the reasons stated therein. These variables are the velocity

$$
v=\sqrt{\frac{2 E}{M}},
$$

where $E$ is the kinetic energy, and the pitch angle $\alpha$. The particle flux is given by

$$
\vec{j}=\int d^{3} v \vec{v} f=\hat{n} J_{\|}+\vec{j}_{\perp} \text {, }
$$

where

$$
\begin{gathered}
J_{\|}=\int d^{3} v v \cos \alpha \bar{f}, \\
\vec{J}_{\perp}=\frac{1}{\Omega \zeta} \hat{n} \times\left[-N \vec{a}+\frac{1}{2} \vec{\nabla}_{\perp}-(\hat{n} \times \vec{\nabla} \times \hat{n})\left(k_{\|}-\frac{1}{2} k_{\perp}\right)\right], \\
N=\int d^{3} v \bar{f}, \\
K_{\perp}=K_{11}+K_{22}=\int d^{3} v v^{2} \sin ^{2} \alpha \bar{f}, \\
K_{\| l}=K_{33}=\int d^{3} v v^{2} \cos ^{2} \alpha \bar{f} .
\end{gathered}
$$


21

The rate of change of flux $(\partial \vec{J} / \partial t)$ is obtained from the above definition of $\vec{j}$ and the appropriate moments of the drift-kinetic equation:

$$
\begin{aligned}
& g_{0}=\left(\frac{\partial J_{\|}}{\partial t}\right)_{0}-(\hat{n} \cdot \vec{a}) N=\int d^{3} v v \cos \alpha \bar{c}-\hat{n} \cdot \overrightarrow{\nabla K} \| \\
& +(\hat{n} \cdot \vec{b})\left(k_{\|}-\frac{1}{2} K_{1}\right) \\
& \vec{g}_{1}=\hat{n}\left(\frac{\partial J_{\|}}{\partial t}\right)_{1}+\frac{\partial \vec{J}_{\perp}}{\partial t} \text {, } \\
& \left(\frac{\partial J_{11}}{\partial t}\right)_{1}=\frac{1}{\Omega} \vec{a} \cdot[\hat{n} \times(\vec{b}-\vec{\nabla})+2 \vec{\nabla} \times \hat{n}-\hat{n}(\hat{n} \cdot \vec{\nabla} \times \hat{n})] J_{\|} \\
& -\frac{1}{5} \vec{\nabla} \cdot\left\{\frac{1}{\Omega}[\vec{\nabla} \times \hat{n}-\hat{n}(\hat{n} \cdot \vec{\nabla} \times \hat{n})][I(1,1)+4 I(3,0)]\right\} \\
& -\frac{1}{5 \Omega}[\hat{n} \times \vec{b}+2 \vec{\nabla} \times \hat{n}-\hat{n}(\hat{n} \cdot \vec{\nabla} \times \hat{n})] \cdot \vec{\nabla}[I(1,1)-I(3,0)], \\
& \frac{\partial \vec{J}_{\perp}}{\partial t}=\frac{1}{\Omega} \hat{n} \times\left\{-N \frac{\partial \vec{a}}{\partial t}+\vec{\nabla} \cdot\left(\hat{\hat{n}}_{\|}\right) \vec{a}+\frac{1}{2} \vec{\nabla}\left(\frac{\partial K_{\perp}}{\partial t}\right)_{0}\right. \\
& \left.-(\hat{n} \times \vec{\nabla} \times \hat{n})\left[\left(\frac{\partial K_{\|}}{\partial t}\right)_{0}-\frac{1}{2}\left(\frac{\partial K_{\perp}}{\partial t}\right)_{0}\right]\right\},
\end{aligned}
$$

where

$$
I(l, q)=\int d^{3} v v^{\ell+2 q} P_{\ell}(\cos \alpha) \bar{f}
$$

$$
\begin{aligned}
\left(\frac{\partial K_{\perp}}{\partial \vec{t}}\right)_{0}= & \int d^{3} v v^{2} \sin ^{2} \alpha \bar{c}+\frac{4}{5}(\hat{n} \cdot \vec{b})[I(1,1)-I(3,0)] \\
& -\frac{2}{5} \hat{n} \cdot \vec{\nabla}[I(1,1)-I(3,0)], \\
& \left(\frac{\partial K_{\|}}{\partial t}\right)_{0}=\int d^{3} v v^{2} \cos ^{2} \alpha \bar{c}+2(\hat{n} \cdot \vec{a}) J_{\|} .
\end{aligned}
$$




$$
+\frac{1}{5}(\hat{n} \cdot \vec{b})[I(1,1)+4 I(3,0)]-\frac{1}{5} \hat{n} \cdot \vec{\nabla}[3 I(1,1)+2 I(3,0)] .
$$

It is to be noted that $g_{0}$ consists of readily identifiable terms: the parallel collisional friction term, the parallel gradient of the parallel energy density or pressure, and a tensor-pressure term proportional to the parallel gradient of the magnetic field. 
INTERNAL DISTRIBUTION

ORNL/TM-5784

1. J. D. Callen

2. J. F. Clarke

3. R. A. Dory

4. G. G. Kelley

5. 0. B. Morgan

6. M. W. Rosenthal

7-31. C. 0. Beasley, Jr.

32-34. Laboratory Records

35. Laboratory Records, ORNL - RC

36. $Y-12$ Document Reference Section

37-38. Central Research Library

39. Fusion Energy Division Library

40. Fusion Energy Division Reports Office

41. ORNL Patent Office

\section{EXTERNAL DISTRIBUTION}

42. Plasma Physics Library, Plasma Physics Laboratory, Princeton Univ., Forrestal Campus, P.0. Box 451, Princeton, NJ 08540

43. Controlled Thermonuclear Research Library, Lawrence Livermore Laboratory, P.0. Box 808, Livermore, CA 94550

44. Q Division Library, c/o F. L. Ribe, Los Alamos Scientific Laboratory, P.0. Box 1663, Los Alamos, NM 87544

45. Controlled Thermonuclear Research Library, c/o Weston M. Stacey, Jr., Argonne National Laboratory, 9700 S. Cass Ave., Argonne, IL 60439

46. CTR Computer Center, c/o Dr. John Killeen, Lawrence Livermore Laboratory, P.0. Box 808, Livermore, CA 94550

47. Librarian, Culham Laboratory, U.K. Atomic Energy Authority, Abingdon, Oxon, OX14 3DB, United Kingdom

48. Ruth Lengye, Bibliothek, Max-Planck Institut für Plasmaphysik, 8046 Garching bei München, Federal Republic of Germany

49. Library, Centre de Recherches en Physique des Plasmas, 21 Avenue des Bains, 1007, Lausanne, Switzerland

50. A. M. Dupas, Documentation S.I.G.N., Départment de la Physique du Plasma et.de la Fusion Controlée, Association EURATOM-CEA sur la Fusion, Centre d'Etudes Nucléaires, BP 85 Centre Du TRI 38041 Grenoble Cedex (France)

51. Bibliotheque, Service du Confinement des Plasmas, C.E.A., B.P. No. 6, 92, Fonteriay-aux-Roses (Seine) France 
52. Library, International Centre for Theoretical Physics, Trieste, Italy

53. Library, Laboratorio Gas Ionizzati, Frascati, Italy

54. V. E. Ivanov, Physical-Technical Institute of the Ukranian Academy of Sciences, Sukhumi, U.S.S.R.

55. L. M. Kovrizhnikh, Lebedev Institute of Physics, Academy of Sciences of the U.S.S.R., Leninsky Prospect 53, Moscow, U.S.S.R.

56. Thermonuclear Laboratory, Kurchatov Institute of Atomic Energy, 46 Ulitsa Kurchatova, P.0. Box 3402, Moscow, U.S.S.R.

57. Library, Institute for Plasma Physics, Nagoya Univ., Nagoya, Japan 464

58. Library, FOM-Institut voor Plasma-Fysica, Rijnhuizen, Jutphaas, Netherlands

59. Plasma Physics Group, Department of Engineering Physics, Australian National University, P.0. Box 4, Canberra A.C.T. 2600 Australia

60. Thermonuclear Library, Japan Atomic Energy Research Institute, Tokai, Naka, Ibaraki, Japan

61. Or. D. G. McAlees, Exxon Nuclear Co., Inc., Research \& Technology Laser Enrichment Department, 2955 George Washington Way, Richland, WA 99352

62. CIR Reading Room, c/o Prof. D. W. Kerst, Dept. of Physics, Sterling Hal1, Univ. of Wisconsin, Madison, WI 53706.

63. CTR Reading Room, c/o Prof. I. B. Bernstein, Yale Univ., New Haven, CT 06510

64. Center for Plasma Physics and Thermonuclear Research,.$/ 00$. W. Ross, Physics Dept., Univ. of Texas, Austin, TX 78712

65. CTR Reading Room, c/o Prof. B. D. Fried, Physics Dept., Univ. of California, Los Angeles, CA 90021

66. CTR Reading Room, c/o Prof. David C. Montgomery, Physics \& Astronomy Dept., Univ. of Iowa, Iowa City, IA 52240

67. Magneto-Fluid-Dynamics Library, Courant Inst. of Math. Sci., New York Univ., 251 Mercer St., New York, NY 10012

68. CTR Reading Room, c/o Prof. Allan N. Kaufman, Physics Dept., Univ. of California, Berkeley, CA 94720

69. CTR Reading Room, c/o Prof. W. B. Thompson, Physics Dept., Univ. of California, San Diego, La Jolla, CA 92037 
70. CTR Reading Room, c/o Prof. Alvin W. Trivelpiece, Dept. of Physics \& Astronomy, Univ. of Maryland, College Park, MD 20742

71. CTR Reading Room, c/o Prof. T. Kammash, 103 Research Admin. Bldg., N. Campus, Univ. of Michigan, Ann Arbor, MI 48105

72. CTR Reading Room, c/o Dr. Ravi N. Sudan, Phillips Hall, Cornell Univ., I thaca, NY 14850

73. Prof. Marshall N. Rosenbluth, Institute for Advanced Study, Princeton, NJ 08540

74. CTR Reading Room, c/o Prof. R. Gross, Plasma Research Lab., Columbia Univ., New York, NY 10027

75. CTR Reading Room, c/o Prof. Roy Gould, California Inst. of Tech., M.S. 116-81, Pasadena, CA 91125

76. Dr. Nicholas A. Krall, Science Applications, Inc., P.0. Box 2354, 1200 Prospect St., La Jolla, CA 92037

77. CTR Reading Room, c/o Dr. Jay P. Boris, Plasma Physics, Naval Research Laboratory, Washington, DC 20390

78. Professor A. Simon, Dept. of Mechanical \& Aerospace Sciences, University of Rochester, Rochester, NY 14627

79. CTR Library, c/o Dr. Alan F. Haught, United Technologies Research Labs, East Hartford, CT 06108

80. Dr. Robert E. Price, Division of Magnetic Fusion Energy, G-234, Energy Research and Development Administration, Washington, DC 20545

81. Dr. R. C. Davidson, Division of Magnetic Fusion Energy, G-234, Energy Research and Development Administration, Washington, DC 20545

82. Dr. Arthur Sleeper, Division of Magnetic Fusion Energy, G-234, Energy Research and Development Administration, Washington, DC 20545

83. Dr. Oscar P. Manley, Division of Magnetic Fusion Energy, G-234, Energy Research and Development Administration, Washington, $D C$ 20545

84. Dr. D. H. Priester, Division of Magnetic Fusion Energy, G-234, Energy Research and Development Administration, Washington, $D C$ 20545

85. Mr. E. E. Kintner, Division of Magnetic Fusion Energy, G-234, Energy Research and Development Administration, Washington, DC 20545 
86. Dr. L. D. Pearlstein, L-388, Lawrence Livermore Laboratory, P.0. Box 808, Livermore, CA 94550

87. Dr. J. P. Friedberg, Los Alamos Scientific Laboratory, Los Alamos NM 87544

88. Dr. David J. Rose, Dept. of Nuclear Engineering, MIT, Cambridge, MA 02139

89. Dr. Gareth E. Guest, General Atomic Co., P.0. Box 81608, San Diego, CA 92138

90. Dr. Claude Mercier, Service du Theorie des Plasmas, Centre d'Etudes Nucleáires, Fontenay-aux-Roses (Seine) France

91. Dr. J. B. Taylor, Culham Laboratory, UKAEA Abingdon, Oxon, Ox14 3DB, United Kingdom

92. Dr. D. Pfirsch, Institute for Plasma Physics, 8046 Garching bei München, Federal Republic of Germany

93. Dr. V. D. Shafranov, I. V. Kurchatov Inst. of Atomic Energy, 46 Ulitsa Kurchatova, P.0. Box 3402, Moscow, U.S.S.R.

94. Dr. Harold Grad, Courant Inst. of Math. Sci., New York Univ., 251 Mercer St., New York, NY 10012

95. Dr. J. G. Cordey, Cul ham Laboratory, UKAEA, Abingdon, 0xon, 0X14 $3 \mathrm{nB}$, United Kingdom

96. Dr. David Baldwin, L-388, Lawrence Livermore Lab., P. . Rnx 808 , Livermore, CA 94550

97. CTR Reading Room, c/o Prof. Bruno Coppi, Dept. of Physics, MIT, Cambridge, MA 02139

98. Dr. Harold P. Furth, Princeton Plasma Physics Lab., Princeton Univ., P.0. Box 451, Princeton, NJ 08540

99. Dr. Paul H. Rutherford. Princet.nn Plasma Physics Lab., Princeton Univ., Princeton, NJ 08540

100. Dr. Herbert H. Woodson, Dept. of Electrical Engineering, Univ. of Texas, Aust1n, TX 78712

101. Research \& Technical Support. Div., Oak Ridge Operations, Energy Research and Development Administration, P. O. Box E, Oak Ridge, TN 37830

102- Tech. Information Center, Oak Ridge Operations, Energy Research

128. and Development Administration, P. 0. Box 62, Oak Ridge, TN 37830 